\title{
ON A DIRICHLET SERIES ASSOCIATED WITH A POLYNOMIAL
}

\author{
MINKING EIE
}

(Communicated by William Adams)

\begin{abstract}
Let $P(x)=\prod_{j=1}^{k}\left(x+\delta_{j}\right)$ be a polynomial with real coefficients and $\operatorname{Re} \delta_{j}>-1 \quad(j=1, \ldots, k)$. Define the zeta function $Z_{P}(s)$ associated with the polynomial $P(x)$ as

$$
Z_{P}(s)=\sum_{n=1}^{\infty} \frac{1}{P(n)^{s}}, \quad \operatorname{Re} s>1 / k
$$

$Z_{P}(s)$ is holomorphic for $\operatorname{Re} s>1 / k$ and it has an analytic continuation in the whole complex $s$-plane with only possible simple poles at $s=j / k \quad(j=$ $1,0,-1,-2,-3, \ldots)$ other than nonpositive integers.

In this paper, we shall obtain the explicit value of $Z_{P}(-m)$ for any nonnegative integer $m$, the asymptotic formula of $Z_{P}(s)$ at $s=1 / k$, the value $Z_{P}^{\prime}(0)$ and its application to the determinants of elliptic operators.
\end{abstract}

\section{INTRODUCTION}

Let $k$ be a positive integer and $P(x)=\prod_{j=1}^{k}\left(x+\delta_{j}\right)$ be a polynomial with real coefficients and $\operatorname{Re} \delta_{j}>-1(j=1, \ldots, k)$. Define $Z_{P}(s)=$ $\sum_{n=1}^{\infty} 1 / P(n)^{s}, \operatorname{Re} s>1 / k$. An elementary consideration shows that $Z_{P}(s)$ is holomorphic for $\operatorname{Re} s>1 / k$. As we shall see soon, $Z_{P}(s)$ has an analytic continuation in the whole complex plane, which has only possible simple poles at $s=j / k(j=1,0,-1,-2,-3, \ldots)$ other than nonpositive integers.

Here we shall prove the following explicit formula for the values of $Z_{P}(s)$ at nonpositive integers and the value $Z_{P}^{\prime}(0)$.

Main Theorem I. $Z_{P}(s)$ has an analytic continuation in the complex which is holomorphic except possible simple poles at $s=j / k(j \leq 1, j$ not a multiple of $k$ ). Furthermore, for any nonnegative integer $m$, we have

$$
Z_{P}(-m)=\sum_{r=0}^{k m} C_{r}\left(\delta_{1}, \ldots, \delta_{k}\right) \frac{(-1)^{r} B_{r+1}}{r+1}+\frac{1}{k} \sum_{j=1}^{k} \int_{0}^{-\delta j} P(x)^{m} d x,
$$

Received by the editors June 3, 1989 and, in revised form, January 26, 1990.

1980 Mathematics Subject Classification (1985 Revision). Primary 11M06.

This work was supported by the Institute of Mathematics, Academia Sinica, Taipei, Taiwan, NSF of Taiwan, Republic of China and the Department of Mathematics, University of Chicago. 
where the coefficients $C_{r}\left(\delta_{1}, \ldots, \delta_{k}\right)(r=0, \ldots, k m)$ are determined by the multinomial expansion $\sum_{r=0}^{k m} C_{r}\left(\delta_{1}, \ldots, \delta_{k}\right) x^{r}=[P(x)]^{m}$ and $B_{r}(r=0,1,2$, $3 . .$.$) are Bernoulli numbers defined by$

$$
\frac{t}{e^{t}-1}=\sum_{j=0}^{\infty} \frac{B_{i} t^{j}}{j !}, \quad|t|<2 \pi .
$$

Main Theorem II. For $\left|\delta_{j}\right|<1 \quad(j=1, \ldots, k)$, we have

$$
Z_{P}^{\prime}(0)=-\frac{k}{2} \log 2 \pi+\sum_{j=1}^{k} \log \Gamma\left(1+\delta_{j}\right) .
$$

The value $Z_{P}^{\prime}(0)$ is useful in the computation of determinants of elliptic operators. Consider the Hardy space of boundary values of $L^{2}$-holomorphic function defined on the unit disc, module constant functions, $H=\left\{\left.\sum_{n=1}^{\infty} a_{n} z^{n}\left|\sum_{n=1}^{\infty}\right| a_{n}\right|^{2}\right.$ $<\infty\}$. Let $D=\frac{1}{i} \frac{d}{d \theta}$. Then $P(D)$ is an elliptic operator and its determinant is defined as $\operatorname{det} P(D)=e^{-Z_{P}^{\prime}(0)}$. With our assertion, we conclude that $\operatorname{det} P(D)=(2 \pi)^{k / 2}\left[\prod_{j=1}^{k} \Gamma\left(1+\delta_{j}\right)\right]^{-1}$.

\section{The analytic CONTINUATION of $Z_{P}(s)$}

For our convenience, we consider the cases $k \geq 2$. However, with a modification if necessary, our result works even for $k=1$. The proof of the main theorem depends on the following proposition.

Proposition 1. For $k \geq 2$ and $\operatorname{Re} s>1 / k$, we have

$$
Z_{P}(s)[\Gamma(s)]^{k}=\int_{0}^{\infty} \frac{t^{k s-1}}{e^{t}-1} d t \int_{E}\left(u_{1} \cdots u_{k}\right)^{s-1} e^{-\left(\delta_{1} u_{1}+\cdots+\delta_{k} u_{k}\right) t} d u_{1} \cdots d u_{k-1},
$$

where $E$ is the standard simplex in $\mathbf{R}^{k-1}$ defined by $u_{1}, \ldots, u_{k-1} \geq 0 ; u_{k}=$ $1-u_{1}-\cdots-u_{k-1} \geq 0$.

Proof. For $\operatorname{Re} \delta_{j}>-1$, we have

$$
\int_{0}^{\infty} y_{j}^{s-1} \exp \left\{-\left(n+\delta_{j}\right) y_{j}\right\} d y_{j}=\left(n+\delta_{j}\right)^{-s} \Gamma(s), \quad j=1, \ldots, k .
$$

Multiply together and let $n$ range over all positive integers. We get

$$
\begin{aligned}
& Z_{P}(s)[\Gamma(s)]^{k}=\sum_{n=1}^{\infty} P(n)^{-s}[\Gamma(s)]^{k}=\sum_{n=1}^{\infty}\left(\prod_{j=1}^{k} \int_{0}^{\infty} y_{j}^{s-1} \exp \left\{-\left(n+\delta_{j}\right) y_{j}\right\} d y_{j}\right) \\
& =\int_{0}^{\infty} \cdots \int_{0}^{\infty}\left(y_{1} \cdots y_{k}\right)^{s-1} \sum_{n=1}^{\infty} \exp \left\{-\left(n+\delta_{1}\right) y_{1}-\cdots-\left(n+\delta_{k}\right) y_{k}\right\} d y_{1} \cdots d y_{k} \\
& =\int_{0}^{\infty} \cdots \int_{0}^{\infty} \frac{\left(y_{1} \cdots y_{k}\right)^{s-1} \exp \left\{-\delta_{1} y_{1}-\cdots-\delta_{k} y_{k}\right\} d y_{1} \cdots d y_{k}}{e^{y_{1}+\cdots+y_{k}}-1} .
\end{aligned}
$$


Now apply the following change of variables to $y_{k-1}$ and $y_{k}$ :

$$
\left\{\begin{array}{l}
y_{k-1}=t_{k-1} t_{k} \\
y_{k}=t_{k-1}\left(1-t_{k}\right)
\end{array}\right.
$$

Then $0<t_{k-1}<\infty$ and $0 \leq t_{k} \leq 1$. Again apply the same change of variables to $y_{k-2}, t_{k-1}$ and continue in this way. We get

$$
\begin{aligned}
& Z_{P}(s)[\Gamma(s)]^{k} \\
& \quad=\int_{0}^{\infty} \frac{t_{1}^{k s-1}}{e^{t_{1}}-1} d t_{1} \int_{0}^{1}\left(t_{2}\right)^{s-1}\left(1-t_{2}\right)^{(k-1) s-1} d t_{2} \cdots \int_{0}^{1}\left[t_{k}\left(1-t_{k}\right)\right]^{s-1} G\left(t_{1}, t_{2}, \ldots, t_{k}\right) d t_{k}, \\
& \text { with } \\
& \quad \begin{array}{l}
G\left(t_{1}, \ldots, t_{k}\right) \\
\quad=\exp \left\{-t_{1}\left[\delta_{1} t_{2}+\delta_{2}\left(1-t_{2}\right)+\cdots+\delta_{k-1} t_{k}\left(1-t_{2}\right) \cdots\left(1-t_{k-1}\right)+\delta_{k}\left(1-t_{2}\right) \cdots\left(1-t_{k}\right)\right]\right\}
\end{array}
\end{aligned}
$$

Finally, we let $t=t_{1}, u_{1}=t_{2}, \ldots, u_{k-1}=t_{k}\left(1-t_{2}\right) \cdots\left(1-t_{k-1}\right)$. Then $u_{k}=1-u_{1}-\cdots-u_{k-1}=\left(1-t_{2}\right) \cdots\left(1-t_{k-1}\right)\left(1-t_{k}\right)$ and $G\left(t_{1}, t_{2}, \ldots, t_{k}\right)$ is transformed into $\exp \left\{-t\left(\delta_{1} u_{1}+\cdots+\delta_{k} u_{k}\right)\right\}$. Also our integral expression for $Z_{P}(s)[\Gamma(s)]^{k}$ is transformed into

$$
\int_{0}^{\infty} \frac{t^{k s-1}}{e^{t}-1} d t \int_{E}\left(u_{1} \cdots u_{k}\right)^{s-1} e^{-t\left(\delta_{1} u_{1}+\cdots+\delta_{k} u_{k}\right)} d u_{1} \cdots d u_{k-1} .
$$

This proves our assertion.

\section{The PROOF of MAIN TheOREM I}

We divide the proof of Main Theorem I into two steps:

Step I. The analytic continuation of $Z_{P}(s)$. For $\operatorname{Re} s>0$, set

$$
I(s, t)=\frac{1}{\Gamma(s)^{k-1}} \int_{E}\left(u_{1} \cdots u_{k}\right)^{s-1} e^{-t\left(\delta_{1} u_{1}+\cdots+\delta_{k} u_{k}\right)} d u_{1} \cdots d u_{k-1} .
$$

As a function of $s, I(s, t)$ is a holomorphic function since the integration is absolutely convergent for $\operatorname{Re} s \geq \varepsilon>0$. Expanding the function

$$
\exp \left\{-t\left(\delta_{1} u_{1}+\cdots+\delta_{k} u_{k}\right)\right\}
$$

as a power series in $t$ and performing a term-by-term integration, we get

$$
I(s, t)=\sum_{j=0}^{\infty}\left[\sum_{p_{1}+\cdots+p_{k}=j} \frac{\Gamma\left(s+p_{1}\right) \cdots \Gamma\left(s+p_{k}\right)(-1)^{j} \delta_{1}^{p_{1}} \cdots \delta_{k}^{p_{k}}}{\Gamma(s)^{k-1} \Gamma(k s+j)\left(p_{1} !\right) \cdots\left(p_{k} !\right)}\right] t^{j}
$$

by using the formula

$$
\int_{E}\left(u_{1}^{s+p_{1}-1} \cdots u_{k}^{s+p_{k}-1}\right) d u_{1} \cdots d u_{k-1}=\frac{\Gamma\left(s+p_{1}\right) \cdots \Gamma\left(s+p_{k}\right)}{\Gamma\left(k s+p_{1}+\cdots+p_{k}\right)} .
$$

Let

$$
A_{j, p}(s)=\frac{\Gamma\left(s+p_{1}\right) \cdots \Gamma\left(s+p_{k}\right)}{\Gamma(s)^{k-1} \Gamma(k s+j)}, \quad p_{1}+\cdots+p_{k}=j .
$$


$A_{j, p}(s)$ is a meromorphic function in $s$ with possible poles at $s=0,-1,-2$, $\ldots,-n, \ldots$. Here we prove that $A_{j, p}(s)$ is also analytic at these points. Let $s=-m$ with $m$ a positive integer or zero. Then we have the following two cases.

Case 1. Suppose $0 \leq p_{1}, \ldots, p_{k} \leq m$. Then $\Gamma\left(s+p_{1}\right) \cdots \Gamma\left(s+p_{k}\right)$ has a pole of order $k$ at $s=-m$. But $j=p_{1}+\cdots+p_{k} \leq k m$, so it follows that $\Gamma(s)^{k-1} \Gamma(k s+j)$ also has a pole of order $k$ at $s=-m$. So the ratio of these two functions, $A_{j, p}(s)$, is analytic at $s=-m$.

Case 2. Suppose $p_{j} \geq m+1$ for some $j$. Then the order of the pole of $\Gamma\left(s+p_{1}\right) \cdots \Gamma\left(s+p_{k}\right)$ (at most $\left.k-1\right)$ is no more than the order of the pole of $\Gamma(s)^{k-1} \Gamma(k s+j)($ at least $k-1)$ at $s=-m$. Hence $A_{j, p}(s)$ is also analytic at $s=-m$.

Consequently, $I(s, t)$, as a function of $s$, has its analytic continuation for all $s$. Furthermore, it is an entire function of $s$. From the formula

(A)

$$
\begin{aligned}
Z_{P}(s)[\Gamma(s)]= & \int_{0}^{\infty} \frac{t^{k s-1} I(s, t) d t}{e^{t}-1} \\
= & \int_{0}^{1} t^{k s-2}\left[\sum_{r=0}^{\infty} \frac{B_{r} t^{r}}{r !}\right]\left[\sum_{j=0}^{\infty} t^{j} \sum_{p_{1}+\cdots+p_{k}=j}(-1)^{j} A_{j, p}(s) \frac{\delta_{1}^{p_{1}} \cdots \delta_{k}^{p_{k}}}{p_{1} ! \cdots p_{k} !}\right] d t \\
& +\int_{1}^{\infty} \frac{t^{k s-1} I(s, t) d t}{e^{t}-1},
\end{aligned}
$$

we see that $Z_{P}(s) \Gamma(s)$ has an analytic continuation which is holomorphic except possible simple poles at $s=j / k(j=1,0,-1,-2,-3, \ldots)$. But, on the other hand, $\Gamma(s)$ has simple poles at $s=0$ as well as at negative integers. Thus the analytic continuation of $Z_{P}(s)$ given by

$$
\frac{1}{\Gamma(s)} \int_{0}^{\infty} \frac{t^{k s-1} I(s, t)}{e^{t}-1} d t
$$

has only possible simple poles at $s=j / k(j=1,0,-1,-2,-3, \ldots)$ other than nonpositive integers.

Step II. The calculation of the value $Z_{P}(-m)$. To determine the value of $Z_{P}(-m)$, we simply compute the residue of $Z_{P}(s) \Gamma(s)$ at $s=-m$. On one hand, the residue of $Z_{P}(s) \Gamma(s)$ at $s=-m$ is given by $(-1)^{m} Z_{P}(-m) / m !$. On the other hand, from formula (A), the residue of $Z_{P}(s) \Gamma(s)$ at $s=-m$ is also given by

It follows that

$$
\frac{1}{k} \sum_{r+j=k m+1} \frac{B_{r}}{r !}\left[\sum_{p_{1}+\cdots+p_{k}=j}(-1)^{j} A_{j, p}(-m) \frac{\delta_{1}^{p_{1}} \cdots \delta_{k}^{p_{k}}}{p_{1} ! \cdots p_{k} !}\right] .
$$

$$
Z_{p}(-m)=\frac{(-1)^{m} m !}{k} \sum_{r+j=k m+1} \frac{B_{r}}{r !}\left[\sum_{p_{1}+\cdots+p_{k}=j}(-1)^{j} A_{j, p}(-m) \frac{\delta_{1}^{p_{1}} \cdots \delta_{k}^{p_{k}}}{p_{1} ! \cdots p_{k} !}\right] .
$$


For any multiple index $p=\left(p_{1}, \ldots, p_{k}\right)$ of $k$-tuple nonnegative integers, define

$$
F_{p}(s)=\frac{\Gamma\left(s+p_{1}\right) \cdots \Gamma\left(s+p_{k}\right)}{\Gamma(s)^{k-1} \Gamma(k s+|p|)}, \quad|p|=p_{1}+\cdots+p_{k}, \quad p !=\left(p_{1} !\right) \cdots\left(p_{k} !\right) .
$$

Then we can rewrite the formula of $Z_{P}(-m)$ as

$$
Z_{P}(-m)=\frac{(-1)^{m} m !}{k} \sum_{|p| \leq k m+1} \frac{B_{k m+1-|p|} \delta_{1}^{p_{1}} \cdots \delta_{k}^{p_{k}}(-1)^{|p|}}{(k m+1-|p|) ! p !} F_{p}(-m) .
$$

From our definition of $F_{p}(s)$, we see that $F_{p}(-m)=0$ unless $0 \leq p_{1}, \ldots, p_{k} \leq$ $m$ or $|p|=p_{1}+\cdots+p_{k}=k m+1, p_{j} \geq m+1$ for exactly one $j$.

For these two exceptional cases, the value of $F_{p}(-m)$ is given by

$$
F_{p}(-m)= \begin{cases}\frac{(-1)^{(k-1) m}(m !)^{k-1} \cdot k \cdot(k m-|p|) !}{\left(m-p_{1}\right) ! \cdots\left(m-p_{k}\right) !} & \text { if } 0 \leq p_{1}, \ldots, p_{k} \leq m, \\ \frac{(-1)^{|p|-p_{j}}(m !)^{k-1}\left(p_{j}-m-1\right) !}{\left(m-p_{1}\right) ! \cdots\left(m-p_{j-1}\right) !\left(m-p_{j+1}\right) ! \cdots\left(m-p_{k}\right) !} & \text { if }|p|=k m+1, p_{j} \geq m+1 .\end{cases}
$$

Hence

$$
\begin{aligned}
Z_{P}(-m)= & \sum_{0 \leq p_{1}, \ldots, p_{k} \leq m}\left(\begin{array}{c}
m \\
p_{1}
\end{array}\right) \cdots\left(\begin{array}{c}
m \\
p_{k}
\end{array}\right) \frac{(-1)^{k m-|p|} B_{k m+1-|p|}}{k m+1-|p|} \delta_{1}^{p_{1}} \cdots \delta_{k}^{p_{k}} \\
& +\frac{1}{k} \sum_{\substack { j=1 \\
\begin{subarray}{c}{|p|=k m+1 \\
p_{j} \geq m+1{ j = 1 \\
\begin{subarray} { c } { | p | = k m + 1 \\
p _ { j } \geq m + 1 } }\end{subarray}}\left(\begin{array}{c}
m \\
p_{1}
\end{array}\right) \cdots\left(\begin{array}{c}
m \\
p_{j-1}
\end{array}\right)\left(\begin{array}{c}
m \\
p_{j+1}
\end{array}\right) \\
& \cdots\left(\begin{array}{c}
m \\
p_{k}
\end{array}\right) \frac{(-1)^{m-p_{j}} m !\left(p_{j}-m-1\right)}{p_{j} !} \delta_{1}^{p_{1}} \cdots \delta_{k}^{p_{k}} .
\end{aligned}
$$

However,

$$
\begin{gathered}
\sum_{0 \leq p_{1}, \ldots, p_{k} \leq m}\left(\begin{array}{c}
m \\
p_{1}
\end{array}\right) \cdots\left(\begin{array}{c}
m \\
p_{k}
\end{array}\right) x^{k m-|p|} \delta_{1}^{p_{1}} \cdots \delta_{k}^{p_{k}} \\
=\left(x+\delta_{1}\right)^{m} \cdots\left(x+\delta_{k}\right)^{m}=[P(x)]^{m}
\end{gathered}
$$

and the $j$ th term in the second summation is equal to

$$
\begin{aligned}
\int_{0}^{-\delta_{j}}[P(x)]^{m} d x & \\
= & \sum_{0 \leq p_{1}, \ldots, p_{k} \leq m}\left(\begin{array}{c}
m \\
p_{1}
\end{array}\right) \cdots\left(\begin{array}{c}
m \\
p_{j-1}
\end{array}\right)\left(\begin{array}{c}
m \\
p_{j+1}
\end{array}\right) \cdots\left(\begin{array}{c}
m \\
p_{k}
\end{array}\right)\left[\delta_{1}^{p_{1}} \cdots \delta_{j-1}^{p_{j-1}} \delta_{j+1}^{p_{j+1}} \cdots \delta_{k}^{p_{k}}\right] \\
& \cdot \int_{0}^{-\delta_{j}} x^{(k-1) m-|p|+p_{j}}\left(x+\delta_{j}\right)^{m} d x .
\end{aligned}
$$

Finally, we have

$$
Z_{P}(-m)=\sum_{r=0}^{k m} C_{r}\left(\delta_{1}, \ldots, \delta_{k}\right) \frac{(-1)^{r} B_{r+1}}{r+1}+\frac{1}{k} \sum_{j=1}^{k} \int_{0}^{-\delta_{j}}[P(x)]^{m} d x
$$

with $\sum_{r=0}^{k m} C_{r}\left(\delta_{1}, \ldots, \delta_{k}\right) x^{r}=\left(x+\delta_{1}\right)^{m} \cdots\left(x+\delta_{k}\right)^{m}=[P(x)]^{m}$.

This proves Main Theorem I. 


\section{ON THE POWER EXPANSION OF $Z_{P}(s)$}

For $\operatorname{Re} s>1 / k$, we have

$$
Z_{P}(s)[\Gamma(s)]^{k}=\int_{0}^{\infty} \frac{t^{k s-1}}{e^{t}-1} d t \int_{E}\left(u_{1} \cdots u_{k}\right)^{s-1} e^{-t\left(\delta_{1} u_{1}+\cdots+\delta_{k} u_{k}\right)} d u_{1} \cdots d u_{k-1}
$$

Note that the second integration is absolutely convergent for $\operatorname{Re} s>0$ and it has the power series expansion (see page 93 of [2])

$$
\sum_{|\alpha|=0}^{\infty} \frac{(-t)^{|\alpha|} \delta_{1}^{\alpha_{1}} \cdots \delta_{k}^{\alpha_{k}} \Gamma\left(s+\alpha_{1}\right) \cdots \Gamma\left(s+\alpha_{k}\right)}{\alpha_{1} ! \cdots \alpha_{k} ! \Gamma(k s+|\alpha|)}
$$

Also, we have $\int_{0}^{\infty} t^{k s+|\alpha|-1} /\left(e^{t}-1\right) d t=\Gamma(k s+|\alpha|) \zeta(k s+|\alpha|)$ for $\operatorname{Re} s>1 / k$ and $|\alpha| \geq 0$. It follows that

$$
Z_{P}(s)=\sum_{|\alpha|=0}^{\infty} \frac{(-1)^{|\alpha|} \delta_{1}^{\alpha_{1}} \cdots \delta_{k}^{\alpha_{k}} \Gamma\left(s+\alpha_{1}\right) \cdots \Gamma\left(s+\alpha_{k}\right)}{\alpha_{1} ! \cdots \alpha_{k} ![\Gamma(s)]^{k}} \zeta(k s+|\alpha|) .
$$

However, the series on the left-hand side is absolutely convergent for $\left|\delta_{j}\right|<1$ $(j=1, \ldots, k)$ and $\operatorname{Re} s>1 / k$. Hence we prove the following proposition.

Proposition 2. Suppose that $\left|\delta_{j}\right|<1 \quad(j=1, \ldots, k)$ and $\operatorname{Re} s>1 / k$. Then

$$
Z_{P}(s)=\sum_{|\alpha|=0}^{\infty} \frac{(-1)^{|\alpha|} \delta_{1}^{\alpha_{1}} \cdots \delta_{k}^{\alpha_{k}} \Gamma\left(s+\alpha_{1}\right) \cdots \Gamma\left(s+\alpha_{k}\right)}{\alpha_{1} ! \cdots \alpha_{k} ![\Gamma(s)]^{k}} \zeta(k s+|\alpha|) .
$$

From the previous proposition, we obtain the asymptotic expansion of $Z_{P}(s)$ when $\left|\delta_{j}\right|<1 \quad(j=1, \ldots, k)$.

Proposition 3. Suppose $\left|\delta_{j}\right|<1 \quad(j=1, \ldots, k)$. Then the asymptotic formula of $Z_{P}(s)$ at $s=1 / k$ is

$$
Z_{P}(s)=\frac{1}{k} \cdot \frac{1}{s-1 / k}+\gamma_{0}+O\left(s-\frac{1}{k}\right)
$$

with $\gamma_{0}=\lim _{m \rightarrow \infty}\left(\sum_{n=1}^{m} P(n)^{-1 / k}-\log m\right)$.

Proof. Write

$$
Z_{P}(s)=\zeta(k s)+\sum_{|\alpha|=1}^{\infty} \frac{(-1)^{|\alpha|} \delta_{1}^{\alpha_{1}} \cdots \delta_{k}^{\alpha_{k}} \Gamma\left(s+\alpha_{1}\right) \cdots \Gamma\left(s+\alpha_{k}\right)}{\alpha_{1} ! \cdots \alpha_{k} ! \Gamma(s)^{k}} \zeta(k s+|\alpha|) .
$$

The infinite series on the left-hand side is absolutely convergent for $s=1 / k$. The Kronecker limit formula for Riemann zeta function gives $\zeta(k s)=$ $1 /(k s-1)+\gamma+O(s-1 / k)$, where $\gamma$ is the Euler constant defined by

$$
\gamma=\lim _{m \rightarrow \infty}\left(\sum_{n=1}^{m} n^{-1}-\log m\right) \text {. }
$$


It follows that

$$
\gamma_{0}=\gamma+\sum_{|\alpha|=1}^{\infty} \frac{(-1)^{\alpha} \delta_{1}^{\alpha_{1}} \cdots \delta_{k}^{\alpha_{k}} \Gamma\left(1 / k+\alpha_{1}\right) \cdots \Gamma\left(1 / k+\alpha_{k}\right)}{\alpha_{1} ! \cdots \alpha_{k} ! \Gamma(1 / k)^{k}} \zeta(|\alpha|+1) .
$$

For any positive integer $n$,

$$
\begin{aligned}
P(n)^{-1 / k} & =\frac{1}{n}\left(1+\frac{\delta_{1}}{n}\right)^{-1 / k} \cdots\left(1+\frac{\delta_{k}}{n}\right)^{-1 / k} \\
& =\frac{1}{n} \sum_{|\alpha|=0}^{\infty} \frac{(-1)^{\alpha} \delta_{1}^{\alpha_{1}} \cdots \delta_{k}^{\alpha_{k}} \Gamma\left(1 / k+\alpha_{1}\right) \cdots \Gamma\left(1 / k+\alpha_{k}\right)}{\alpha_{1} ! \cdots \alpha_{k} ! \Gamma(1 / k)^{k}} n^{-|\alpha|} .
\end{aligned}
$$

Hence

$$
\begin{aligned}
\lim _{m \rightarrow \infty} & \left\{\sum_{n=1}^{m}\left[P(n)^{-1 / k}-\frac{1}{n}\right]\right\} \\
= & \sum_{|\alpha|=1}^{\infty} \frac{(-1)^{\alpha} \delta_{1}^{\alpha_{1}} \cdots \delta_{k}^{\alpha_{k}} \Gamma\left(1 / k+\alpha_{1}\right) \cdots \Gamma\left(1 / k+\alpha_{k}\right)}{\alpha_{1} ! \cdots \alpha_{k} ! \Gamma(1 / k)^{k}} \zeta(|\alpha|+1) .
\end{aligned}
$$

Consequently, we have

$$
\begin{aligned}
\gamma_{0} & =\lim _{m \rightarrow \infty}\left(\sum_{n=1}^{m} P(n)^{-1 / k}-\sum_{n=1}^{m} \frac{1}{n}\right)+\gamma \\
& =\lim _{m \rightarrow \infty}\left(\sum_{n=1}^{m} P(n)^{-1 / k}-\log m\right)-\gamma+\gamma \\
& =\lim _{m \rightarrow \infty}\left(\sum_{n=1}^{m} P(n)^{-1 / k}-\log m\right) .
\end{aligned}
$$

\section{The PRoOF OF MAIN TheOREM II}

The series expression

$$
Z_{P}(s)=\sum_{|\alpha|=0}^{\infty} \frac{(-1)^{|\alpha|} \delta_{1}^{\alpha_{1}} \cdots \delta_{k}^{\alpha_{k}} \Gamma\left(s+\alpha_{1}\right) \cdots \Gamma\left(s+\alpha_{k}\right)}{\alpha_{1} ! \cdots \alpha_{k} ! \Gamma(s)^{k}} \zeta(k s+|\alpha|)
$$

works not only for $\operatorname{Re} s>1 / k$, but also for all $s$ when $\left|\delta_{j}\right|<1(j=1,2$, $\ldots, k)$. Indeed, for any $s=\sigma+i \tau$, the subseries

$$
\sum_{2 \leq \alpha_{1}+\sigma} \cdots \sum_{2 \leq \alpha_{k}+\sigma} \frac{(-1)^{|\alpha|} \delta_{1}^{\alpha_{1}} \cdots \delta_{k}^{\alpha_{k}} \Gamma\left(s+\alpha_{1}\right) \cdots \Gamma\left(s+\alpha_{k}\right)}{\alpha_{1} ! \cdots \alpha_{k} ![\Gamma(s)]^{k}} \zeta(k s+|\alpha|)
$$

is absolutely convergent. With this observation, we are ready to compute the value $Z_{P}^{\prime}(0)$. 
The proof of Main Theorem II. Rewrite $Z_{P}(s)$ as

$$
\begin{aligned}
Z_{P}(s)= & \zeta(k s)-s\left(\delta_{1}+\cdots+\delta_{k}\right) \zeta(k s+1) \\
& +\sum_{|\alpha|=2}^{\infty} \frac{(-1)^{|\alpha|} \delta_{1}^{\alpha_{1}} \cdots \delta_{k}^{\alpha_{k}} \Gamma\left(s+\alpha_{1}\right) \cdots \Gamma\left(s+\alpha_{k}\right)}{\alpha_{1} ! \cdots \alpha_{k} ![\Gamma(s)]^{k}} \zeta(k s+|\alpha|) .
\end{aligned}
$$

Let $Z_{P}^{*}(s)$ be the series on the left-hand side. Then

$$
Z_{P}^{*}(s)=\sum_{|\alpha|=2}^{\infty} \frac{(-1)^{|\alpha|} \delta_{1}^{\alpha_{1}} \cdots \delta_{k}^{\alpha_{k}}}{\alpha_{1} ! \cdots \alpha_{k} !}\left[s \cdot\left(s+\alpha_{1}-1\right)\right] \cdot\left[s \cdots\left(s+\alpha_{k}-1\right)\right] \zeta(k s+|\alpha|) .
$$

The coefficient of $s$ in $Z_{P}^{*}(s)$ at $s=0$ is given by $\sum_{j=1}^{k} \sum_{\alpha_{j}=2}^{\infty}(-1)^{\alpha_{j}} \delta_{j}^{\alpha_{j}} \zeta\left(\alpha_{j}\right) / \alpha_{j}$. It follows that

$$
\begin{aligned}
Z_{P}^{\prime}(0) & =k \zeta^{\prime}(0)-\gamma\left(\delta_{1}+\cdots+\delta_{k}\right)+\sum_{j=1}^{k}\left[\sum_{\alpha_{j}=2}^{\infty} \frac{(-1)^{\alpha_{j}} \delta_{j}^{\alpha_{j}}}{\alpha_{j}} \zeta\left(\alpha_{j}\right)\right] \\
& =-\frac{k}{2} \log 2 \pi+\prod_{j=1}^{k} \log \Gamma\left(1+\delta_{j}\right) .
\end{aligned}
$$

Here we use the well-known formula $\zeta^{\prime}(0)=-\frac{1}{2} \log 2 \pi$ and the Taylor expansion of $\log \Gamma(1+z)$ near $z=0, \log \Gamma(1+z)=-\gamma z+\sum_{n=2}^{\infty}(-z)^{n} \zeta(n) / n$.

\section{REFERENCES}

1. W. L. Baily, Jr., Introductory lectures on automorphic forms, Princeton Univ. Press, 1973.

2. B. C. Carlson, Special functions of applied mathematics, Academic Press, 1977.

3. Minking Eie, A zeta-function associated with zero ternary forms, Proc. Amer. Math. Soc. 94 (1985), 387-392.

4. A. Kurihara, On the values at non-positive integers of Siegel's zeta functions of q-anisotropic quadratic forms with signature $(1, n-1)$, J. Fac. Sci. Univ. Tokyo Sect. IA Math. 28 (1981), 567-584.

5. Y. Namikawa, Toroidal compactification of Siegel spaces, Lecture Notes in Math., vol. 812, Springer-Verlag, Berlin and New York.

6. _ A new compactification of the Siegel space and degeneration of abelian varieties. I, Math. Ann. 221 (1976), 97-141.

7. I. Satake, Special values of zeta functions associated with self dual homogeneous cones, manuscript, 1981.

8. M. Sato and T. Shintani, On zeta functions associated with prehomogeneous vector spaces, Ann. of Math. 100 (1974), 131-170.

9. T. Shintani, Zeta-functions associated with the vector of quadratic forms, J. Fac. Sci. Univ. Tokyo Sect. IA Math. 22 (1975), 25-65.

10. _ On evaluation of zeta functions of totally real algebraic number fields at non-positive integers, J. Fac. Sci. Univ. Tokyo 23 (1976), 393-417. CHINA

Institute of Mathematics, Academia Sinica, Nankan Taipei 11529, Taiwan, Republic of

Current address: Department of Mathematics, University of Chicago, Chicago, Illinois, 60637 